\title{
Role of Angular Electron Pair Correlation in Stabilizing $\mathrm{C}_{60}^{2-}$
}

\author{
SETH DIFLEY, JACK SIMONS \\ Department of Chemistry, Henry Eyring Center for Theoretical Chemistry, University of Utah, \\ Salt Lake City, Utah 84112
}

Received 8 May 2005; accepted 13 June 2005

Published online 7 October 2005 in Wiley InterScience (www.interscience.wiley.com).

DOI 10.1002/qua.20777

\begin{abstract}
A pseudo-potential that was successfully employed in an earlier study by the Compton group is used to describe the binding of a single electron to a $C_{60}$ molecule to form $\mathrm{C}_{60}^{-}$. Then, the interaction of a second electron with the $\mathrm{C}_{60}^{-}$anion is treated in two manners. First, as performed in the earlier Compton study, a mean-field (i.e., Hartree-Fock) approach is used to estimate the $\mathrm{C}_{60}^{-}-$to- $\mathrm{C}_{60}^{2-}$ energy difference for the singlet state of the dianion and, much as in the earlier study, this dianion is predicted to be unstable by $\sim 0.4 \mathrm{eV}$. Second, for this same singlet state, a configuration interaction wave function is employed that allows for the angular correlation of the two excess electrons, allowing them to avoid one another by moving on opposite sides of the $\mathrm{C}_{60}$ skeleton. The energy of the dianion is lowered by $0.3 \mathrm{eV}$ when angular correlation is included, suggesting that the singlet dianion is unstable with respect to electron loss by only $\sim 0.1 \mathrm{eV}$. A Coulomb barrier ( $>1 \mathrm{eV}$ high) and angular momentum barriers then combine to trap electrons of singlet $\mathrm{C}_{60}^{2-}$ from detaching, thus producing the very long observed lifetimes. In addition, the energy of the lowest triplet state of $\mathrm{C}_{60}^{2-}$ is also discussed. (c) 2005 Wiley Periodicals, Inc. Int J Quantum Chem 106: 507-513, 2006
\end{abstract}

Key words: fullerene; correlation; angular correlation; anion; dianion

\section{Introduction}

n 1991, Compton's group [1] reported observing the $\mathrm{C}_{60}^{2-}$ dianion in the gas phase and determined it to be stable to electron auto-detachment for $\geq 10^{-3} \mathrm{~s}$. However, these workers were unable to determine with certainty whether the dianion is electronically

Correspondence to: J. Simons; e-mail: simons@chem.utah.edu Contract grant sponsor: NSF.

Contract grant number: 0240387. stable with respect to $\mathrm{C}_{60}^{-}+e^{-}$or is unstable with its long lifetime resulting from the need for an electron to tunnel through a barrier to undergo detachment. In 1997, Compton et al. [2] found the larger $C_{84}^{2-}$ dianion to be electronically stable (by $\sim 0.4 \mathrm{eV}$ ) with respect to $\mathrm{C}_{84}^{-}+e^{-}$. Because the two extra electrons in $\mathrm{C}_{60}^{2-}$ are confined to a smaller region of space than those in $\mathrm{C}_{84}^{2-}$, it is likely that the Coulomb repulsions in $\mathrm{C}_{60}^{2-}$ are larger than in $\mathrm{C}_{84}^{2-}$, and thus it is possible that $\mathrm{C}_{60}^{2-}$ could be electronically stable with respect to $\mathrm{C}_{60}^{-}+e^{-}$, but probably by $\leq 0.4 \mathrm{eV}$. 
The theoretical pseudo-potential calculations carried out in Ref. [1] suggested that $\mathrm{C}_{60}^{2-}$ could be bound by $0.1-0.4 \mathrm{eV}$, but the computed second electron binding energy was found to depend strongly on the radius parameter $r_{c}$ used to characterize the size of the spherical $\mathrm{C}_{60}$ molecular skeleton. ${ }^{1}$ In fact, for a value of $r_{c}=5.3 \AA$ (larger than the $\sim 3.5 \AA$ radius nominally assumed [4] to characterize the size of the atomic framework of $\mathrm{C}_{60}, \mathrm{C}_{60}^{2-}$ was predicted to be stable by $0.3 \mathrm{eV}$. In contrast, for $r_{c}=$ $4.2 \AA$, which is still a bit larger than the nominal radius, $\mathrm{C}_{60}^{2-}$ was computed to lie $0.3 \mathrm{eV}$ above $\mathrm{C}_{60}^{-}+$ $e^{-}$. So, unless the sphere used in the pseudo-potential calculations is assumed to be significantly larger than the actual radius of $\mathrm{C}_{60}$, the lowest singlet state of the dianion is predicted to be unstable.

Using both ab initio Hartree-Fock (HF) and density functional theory (DFT) methods and a molecular framework having a radius within the $3.5-\AA$ range, other workers [3-6] concluded that the lowest-energy state of $\mathrm{C}_{60}^{2-}$ is not stable but is unstable by $\sim 0.3 \mathrm{eV}$ relative to $\mathrm{C}_{60}^{-}+e^{-}$. However, these findings cannot be viewed as definitive because (i) the HF treatment ignores the correlated motions of the two "extra" electrons, which we show in this work to be quite stabilizing; and (ii) the DFT treatments suffer, because of the functionals used, from the fact that the exchange correlation potential does not fully cancel the self-interaction appearing in the Coulomb energy functional. As a result, the longrange behavior of the potential experienced by an electron is improperly described; this can be especially problematic for anions and dianions whose charge densities are diffuse.

Although the pseudo-potential [1] and DFT [4] studies may differ on the electronic stability of $\mathrm{C}_{60}^{2-}$, they agree on the fact that this dianion, if electronically unstable, will be rendered long-lived by the presence of a barrier (arising from Coulomb and centrifugal potentials) through which an electron must tunnel to escape. In summary, to the present day it remains undetermined whether the $C_{60}^{2-}$ dianion is stable with respect to its mono-anion and a free electron, but it is well established that, if $\mathrm{C}_{60}^{2-}$ is unstable, it is quite long-lived (with a lifetime of $10^{-3}-0.4 \mathrm{~s}$ ).

Given this state of affairs, it might seem reasonable to attempt a state-of-the-art ab initio calculation of the electronic energies of $C_{60}^{2-}$ and $C_{60}^{-}$. However, a rigorous treatment of all 362 electrons, or even of the 242 valence electrons of the dianion, would be a formidable task. First, a flexible and diffuse atomic orbital basis set would be required.
For example, a triple-zeta plus polarization basis set with one diffuse $s$ and $p$ function per atom, having 22 contracted functions per carbon atom, would involve 1,320 total basis functions. However, even such a basis would probably not produce a sufficiently accurate value for the polarizability of $\mathrm{C}_{60}$, and thus would not accurately represent the most important factor in the electron- $\mathrm{C}_{60}$ interaction potential. Moreover, as illustrated by the results of the pseudo-potential calculations presented in the present study, electron correlation affects would be essential to treat, so calculations would need to be performed, for example, at the second-order Møller-Plesset (MP2) or coupled-cluster level. We note that, although the earlier DFT-level studies [4-6] of $C_{60}^{2-}$ indeed treat some electron correlation effects, they probably do not handle the strong angular correlations that are shown later to contribute significantly for this species. In particular, the kind of correlation operative in $\mathrm{C}_{60}^{2-}$ is very similar to the well-known $2 s^{2} \rightarrow 2 p^{2}$ angular correlation that is prevalent and deemed "essential" in alkaline earth atoms, and which is known to require a multiconfiguration reference wave function.

Our research group has a great deal of experience in studying anions and dianions, using ab initio methods, and it is our opinion, based on the facts just outlined, that a brute-force ab initio treatment of $\mathrm{C}_{60}^{2-}$ and $\mathrm{C}_{60}^{-}$would not, with currently available resources, yield electron-binding energies of the $0.1-0.3-\mathrm{eV}$ accuracy needed to resolve the issue of the stability of $C_{60}^{2}$. We therefore decided to focus our efforts on the specific issue of the extent to which the tendency of the two excess electrons to angularly "avoid" one another due to their Coulomb repulsions will stabilize $\mathrm{C}_{60}^{2-}$ relative to meanfield models such as used in Refs. [1] and [3], where such correlations are ignored. We believe that when highly accurate mean-field calculations on $\mathrm{C}_{60}^{2-}$ become available, the angular correlation energy estimates achieved here can be combined with the mean-field energies to produce more reliable estimates of the stability of this dianion. It seems clear to us that the delocalization of electrons over the $\mathrm{C}_{60}^{2-}$ surface skeleton, combined with the Coulomb repulsions between the two excess charges, should cause these electrons to undergo angularly correlated movements in which the probability density $P\left(\theta_{1}, \phi_{1} ; \theta_{2} ; \phi_{2}\right)$ for one electron residing on the pseudo-spherical $\mathrm{C}_{60}$ framework at angular coordinates $\theta_{1}, \phi_{1}$ when the other is at $\theta_{2}, \phi_{2}$ will be largest when $\theta_{2}=\theta_{1}+\pi$ and $\phi_{2}+\phi_{1}+\pi$. Thus, in this effort, we attempt to estimate the difference be- 
tween the predicted energies of singlet $C_{60}^{2-}$ resulting from a mean-field treatment of the two extra electrons and from a treatment in which angular correlations between these two electrons are incorporated into the quantum wave function.

\section{Methods}

The most essential component of the calculations whose results are under discussion is the choice of the electron $-\mathrm{C}_{60}$ interaction potential. In an ab initio mean-field approach, this potential would be expressed as a sum of electron-nuclear Coulomb attractions (one for each of the 60 carbon nuclei) and electron-electron Coulomb minus exchange potentials, which produce short-range repulsive potentials. The one excess electron of $\mathrm{C}_{60}^{-}$would then be viewed as occupying a low-energy unoccupied molecular orbital of $\mathrm{C}_{60}$ expressed as a linear combination of the $p_{\pi}$ orbitals on the 60 carbon atoms. In Ref. [3], the lowest unoccupied orbital of $C_{60}$ was found to have $t_{1 \mathrm{u}}$ symmetry, so the anion would be expected to have $t_{1 \mathrm{u}}^{1}$ orbital character and the dianion to have $t_{1 u}^{2}$ character. This would suggest that the lowest-energy state of $\mathrm{C}_{60}^{2-}$ could have ${ }^{3} \mathrm{~T}_{1 \mathrm{~g}},{ }^{1} \mathrm{H}_{\mathrm{g}}$ or ${ }^{1} \mathrm{~A}_{\mathrm{g}}$ symmetry. Spin coupling effects (i.e., that induce singlet-triplet energy differences) render the ${ }^{3} \mathrm{~T}_{1 \mathrm{~g}}$ state of $\mathrm{C}_{60}^{2-} \sim 0.5 \mathrm{eV}$ lower than the ${ }^{1} \mathrm{~A}_{\mathrm{g}}$ state. ${ }^{1}$

However, as the original Compton work suggests [1], the nuclear attraction and Coulomb minus exchange potentials discussed above do not represent the dominant long-range contribution (and probably the strongest contribution at all but the very short distances) to the electron- $\mathrm{C}_{60}$ interaction. Rather, it is the charge-induced-dipole polarization potential that is most likely the strongest contributor to the electron binding in $\mathrm{C}_{60}^{-}$. For this reason, rather than beginning with the picture offered by the HF mean-field model as our starting point, we chose to use the Compton model to begin our analysis. In our opinion, the pseudo-potential employed by Compton et al. [1] contains a qualitatively correct and very useful description of the interaction of an electron with a $\mathrm{C}_{60}$ molecule.

\footnotetext{
${ }^{1}$ These calculations assumed that both electrons occupied the same orbital $\Psi(r, \theta, \phi)$ but with opposite spins, so they relate to a singlet-coupled electron pair. It is widely accepted that the lowest-energy unoccupied orbitals of $\mathrm{C}_{60}$ are triply degenerate and of $t_{1 \mathrm{u}}$ symmetry. Placing two electrons into such $t_{1 \mathrm{u}}$ orbitals can result in states of ${ }^{3} \mathrm{~T}_{1 \mathrm{~g}},{ }^{1} \mathrm{H}_{\mathrm{g}}$, and ${ }^{1} \mathrm{~A}_{\mathrm{g}}$ symmetry. According to Ref. [3], the ${ }^{3} \mathrm{~T}_{1 \mathrm{~g}}$ state lies $0.22 \mathrm{eV}$ below the ${ }^{1} \mathrm{H}_{\mathrm{g}}$ state and $0.54 \mathrm{eV}$ below the ${ }^{1} \mathrm{~A}_{\mathrm{g}}$ state.
}

The potential energy describing the interaction of an electron at a distance $r$ from the center of the $\mathrm{C}_{60}$ framework is expressed in this model as

$$
V(r)=-e^{2}(\alpha / 2)\left[c^{2}+\left(r-r_{c}\right)^{2}\right]^{-2} .
$$

This potential has the charge-induced-dipole form $\left[-e^{2} \alpha /\left(2 r^{4}\right)\right]$ at large distances and has a depth of $-e^{2} \alpha /\left(2 c^{4}\right)$ at the nominal surface of the $\mathrm{C}_{60}$ framework $\left(r=r_{c}\right)$. The parameter $\alpha$ is the polarizability of $\mathrm{C}_{60}$ (taken to be $83 \AA^{3}$ ), and $r_{c}$ is the parameter discussed earlier that characterizes the radius of $\mathrm{C}_{60}$. The results shown in Section 3 involve $r_{c}$ ranging from 7 to $10 \mathrm{bohr}(1 \mathrm{bohr}=0.529 \AA$ ) to allow comparisons with the findings of Ref. [1], but we note that 7-8 bohr is most likely to be representative of the true size of $C_{60}$. Finally, as in Ref. [1], for every value of $r_{c}$, parameter $c$, which determines the depth of the electron- $\mathrm{C}_{60}$ potential, is chosen to make the $\mathrm{C}_{60}^{-}$anion stable with respect to $\mathrm{C}_{60}+e^{-}$ by the known experimental detachment energy, $2.65 \mathrm{eV}$ [1]. We note that the ab initio HF calculations reported in Ref. [3] obtained detachment energies of $0.7-0.9 \mathrm{eV}$, providing strong evidence that the polarization potential, which the HF calculation does not include, is crucial.

Given this choice for the electron- $\mathrm{C}_{60}$ potential $V(r)$, we use the following one- and two-electron Hamiltonians to describe the motions of the electron(s) in $\mathrm{C}_{60}^{-}$and $\mathrm{C}_{60}^{2-}$, respectively:

$$
\begin{gathered}
H(1)=-\hbar^{2} / 2 m_{e} \nabla_{1}^{2}+V\left(r_{1}\right) \\
H(1,2)=-\hbar^{2} / 2 m_{e} \nabla_{1}^{2}+V\left(r_{1}\right)-\hbar^{2} / 2 m_{e} \nabla_{2}^{2} \\
+V\left(r_{2}\right)+e^{2} / r_{1,2} .
\end{gathered}
$$

The subscripts 1 and 2 label the two electrons, and $r_{1,2}$ is the distance between them. To obtain a wave function $\Psi(r, \theta, \phi)$ and energy $E=-2.65 \mathrm{eV}$ appropriate for the one-electron $\mathrm{C}_{60}^{-}$, we follow Ref. [1] and assume that the single excess electron is distributed uniformly over the surface of the $\mathrm{C}_{60}$ molecule, so the angular portion of $\psi$ is given by the spherical harmonic $Y_{0,0}(\theta, \phi)$. We note that this wave function is different from what the HF meanfield treatment suggests (i.e., the HF lowest unoccupied molecular orbital [LUMO] has $t_{1 \mathrm{u}}$ symmetry). The radial part of $\Psi$ we denote $F(r) / r$ and we numerically solve the one-dimensional Schrödinger equation resulting from substituting $\Psi(r, \theta, \phi)=Y_{0,0}$ $F / r$ into Eq. (2a) 


$$
-\hbar^{2} / 2 m_{e} d^{2} / d r^{2} F+V(r) F=E F
$$

to obtain $F(r)$, which is subsequently normalized to guarantee that $\Psi$ is normalized. As noted earlier, parameter $c$ is varied to guarantee that the lowest eigenvalue $E$ of Eq. (3) is $-2.65 \mathrm{eV}$.

For the two-electron $\mathrm{C}_{60}^{2-}$, we use two trial variational wave functions of the following forms:

$$
\begin{aligned}
& \Psi_{\mathrm{MF}}(1,2) \\
& \quad=\left|Y_{0,0}\left(\theta_{1}, \phi_{1}\right) F\left(r_{1}\right) / r_{1} \alpha_{1} Y_{0,0}\left(\theta_{2}, \phi_{2}\right) F\left(r_{2}\right) / r_{2} \beta_{2}\right| \\
& \begin{array}{l}
\Psi_{\mathrm{CI} .}(1,2) \\
\quad=A\left|Y_{0,0}\left(\theta_{1}, \phi_{1}\right) F\left(r_{1}\right) / r_{1} \alpha_{1} Y_{0,0}\left(\theta_{2}, \phi_{2}\right) F\left(r_{2}\right) / r_{2} \beta_{2}\right| \\
-B\left|Y_{1,0}\left(\theta_{1}, \phi_{1}\right) F\left(r_{1}\right) / r_{1} \alpha_{1} Y_{1,0}\left(\theta_{2}, \phi_{2}\right) F\left(r_{2}\right) r_{2} \beta_{2}\right| .
\end{array}
\end{aligned}
$$

The mean-field function $\Psi_{\mathrm{MF}}$ consists of a single Slater determinant in which the two electrons are singlet coupled as in Ref. [1]; both are distributed uniformly over the spherical framework, and both are distributed radially in the function $F(r) / r$. Such a wave function allows for no angular or radial correlation between the two excess electrons. The configuration interaction (CI) wave function, ${ }^{2} \Psi_{\mathrm{CI} .}$, also describes two singlet-coupled electrons, but in a way that correlates the angular distributions of the electrons, although it contains no radial correlations because both electrons occupy the same radial space characterized by $F(r) / r$. To see how the angular correlation appears, we note [7] that $\Psi_{\mathrm{CI}}$ can be written in the form

$$
\Psi_{\mathrm{CI}}(1,2)=A / 2\left\{\left|\chi(1) \alpha_{1} \chi^{\prime}(2) \beta_{2}\right|-\left|\chi(1) \beta_{1} \chi^{\prime}(2) \alpha_{2}\right|\right\},
$$

where the so-called polarized orbital pairs, $\chi$ and $\chi^{\prime}$, are defined by

$$
\begin{gathered}
\chi=Y_{0,0} F / r-x Y_{1,0} F / r \\
\chi^{\prime}=Y_{0,0} F / r+x Y_{1,0} F / r
\end{gathered}
$$

\footnotetext{
${ }^{2}$ To form a wave function that has fully symmetric ${ }^{1} \mathrm{~A}$ symmetry, one must, of course, also include terms of the form $\left|Y_{1,1}\left(\theta_{1}, \phi_{1}\right) F\left(r_{1}\right) / r_{1} \alpha_{1} Y_{1,-1}\left(\theta_{2}, \phi_{2}\right) F\left(r_{2}\right) / r_{2} \beta_{2}\right|$ and $\mid Y_{1,-1}\left(\theta_{1}, \phi_{1}\right)$ $F\left(r_{1}\right) / r_{1} \alpha_{1} Y_{1,1}\left(\theta_{2}, \phi_{2}\right) F\left(r_{2}\right) / r_{2} \beta_{2} \mid$. However, for pedagogical purposes, we find it more straightforward to discuss the two-term $\mathrm{CI}$ wave function as written in Eq. (5a). As we will show, even these two terms are adequate to illustrate the concept of angular correlation that we are studying here.
}

and $x=(\mathrm{B} / \mathrm{A})^{1 / 2}$. By expressing $\Psi_{\mathrm{CI}}$ as in Eq. (5), we can see that $\Psi_{\mathrm{CI}}$ describes two singlet-coupled electrons (from the $\alpha \beta-\beta \alpha$ combination of spin functions) that reside in two distinct (nonorthogonal) spatial orbitals, $\chi$ and $\chi^{\prime}$. When one electron is localized in a region of space whose probability density is described by $|\chi|^{2}$, the other electron is localized in the region of space with probability characterized by $\left|\chi^{\prime}\right|^{2}$. The two functions $\chi$ and $\chi^{\prime}$ have angular characters expressed by $\left(Y_{0,0} \mp x Y_{1,0}\right)$; that is, they are $s p$-like hybrid functions whose relative $s$ and $p$ admixture depends on variable $x$ that, in turn, relates to the amplitudes $B$ and $A$ in the CI wave function. If $x$ is small, $\chi$ and $\chi^{\prime}$ are nearly identical, and the two electrons reside in nearly the same regions of space; this, of course, shows how the $C I$ wave function collapses to the mean-field function if $B \ll A$. In contrast, if $x$ is large (i.e., approaching unity), $\chi$ and $\chi^{\prime}$ approach pure $s p$ hybrids, which means that while one electron resides in the $s+p_{z}$ region of space, the other electron is in the $s-p_{z}$ region of space. It is in this manner that the pair of electrons undergo correlated angular movements $^{3}$ very much as the two $2 s$ electrons in the Be atom angularly correlate their movements by using $2 s^{2} \rightarrow 2 p^{2}$ excitations in the CI wave function.

Given the forms for the two wave functions shown in Eqs. (4a) and (4b), we then evaluate the electronic energy of $\mathrm{C}_{60}^{2-}$ in two ways: (i) by computing $\left\langle\Psi_{\mathrm{MF}} \mathrm{H} \mid \Psi_{\mathrm{MF}}\right\rangle$ in the mean-field case, or (ii) by making $\left\langle\Psi_{\mathrm{CI}} \mathrm{H} \mid \Psi_{\mathrm{CI}}\right\rangle$ minimum with respect to the variables $A$ and $B$, keeping $A^{2}+B^{2}=1$ in the CI case.

\section{Results}

Figure 1 shows the radial potentials $V(r)$ of Eq. (1) for four values of the $\mathrm{C}_{60}$ size parameter: $r_{c}=7$, 8,9 , and 10 bohrs (light curves). For each value of $r_{c}$, we also show the radial wave function $F(r)$ obtained by using this $V(r)$ to solve the one-electron Schrödinger Eq. (3). The dark curves in Figure 1 show a potential obtained by adding to $V(r)$ a term $e^{2} /(r+$ $r_{c}$ ) designed to model the Coulomb repulsion experienced by one electron due to the presence of an-

\footnotetext{
${ }^{3}$ If the two other determinants $\mid Y_{1,1}\left(\theta_{1}, \phi_{1}\right) F\left(r_{1}\right) / r_{1} \alpha_{1}$ $Y_{1,-1}\left(\theta_{2}, \phi_{2}\right) F\left(r_{2}\right) / r_{2} \beta_{2} \mid$ and $\mid Y_{1,-1}\left(\theta_{1}, \phi_{1}\right) \quad F\left(r_{1}\right) / r_{1} \alpha_{1} Y_{1,1}\left(\theta_{2}, \phi_{2}\right)$ $F\left(r_{2}\right) / r_{2} \beta_{2} \mid$ had also been included in $\Psi_{\mathrm{CI}}$, we would also have contributions where $\chi$ and $\chi^{\prime}$ involves $s \pm p_{x}$ and $s \pm p_{y}$ polarization. In all cases, the physical picture is one of one electron being on one (angular) side of the $\mathrm{C}_{60}$ framework when the other electron is on the opposite side.
} 

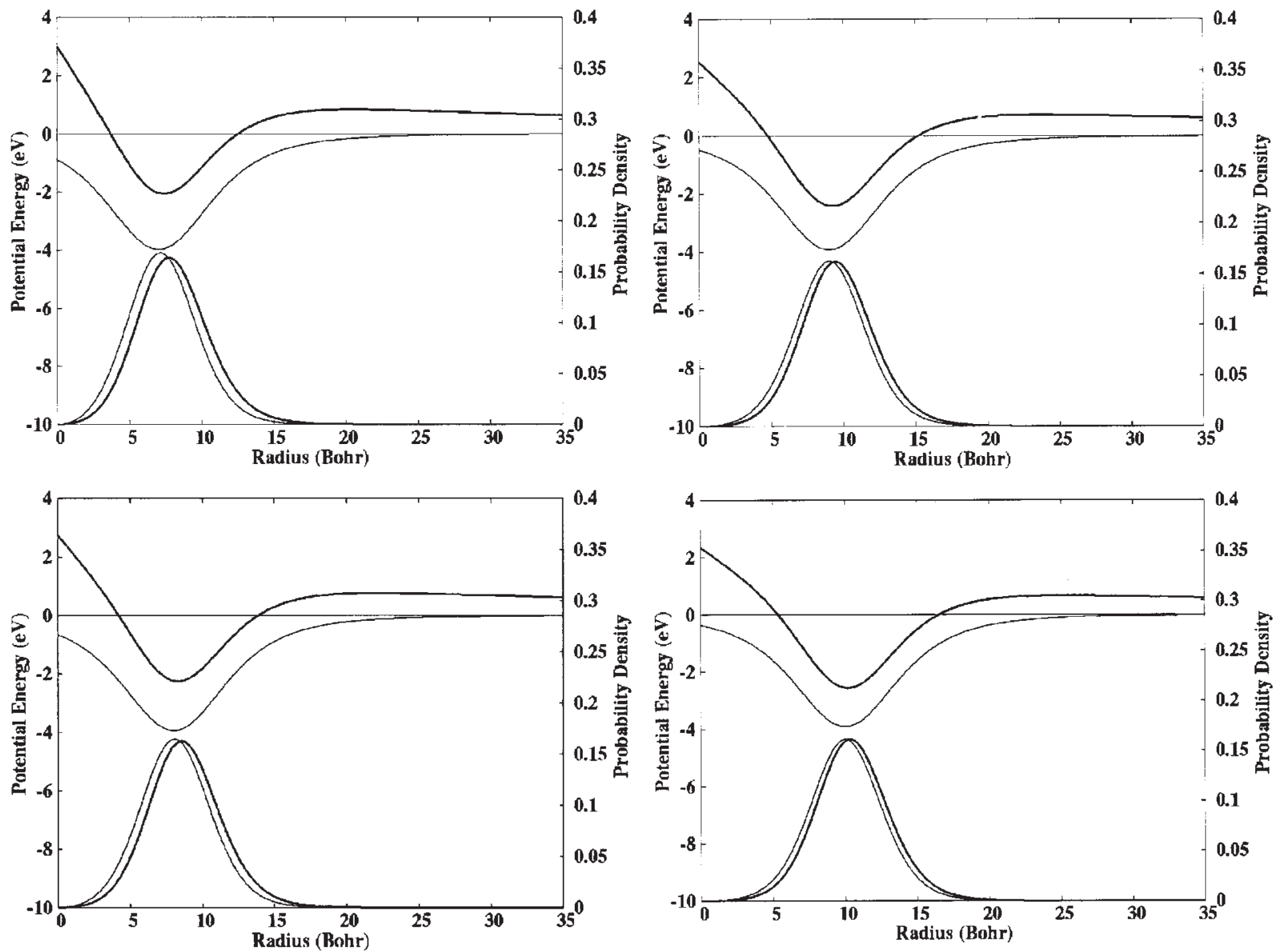

FIGURE 1. Potentials $V(r)$ as expressed in Eq. (1) (light curve, top), potential with repulsive Coulomb potential added to $V(r)$ (dark curve, top) and radial $F(r)$ functions (bottom) resulting from using these two potentials in Eq. (3). The values of $r_{c}$ are $7,8,9$, and 10 bohrs, for the top through bottom figures.

other electron on the opposite side of the $\mathrm{C}_{60}$ molecule. We also show in darker shading the $F(r)$ function that one obtains if one were to use the darker potential in Eq. (3) to solve the one-electron radial Schrödinger equation. It is important to note that we do not make use of the darker potential or its $F(r)$ in arriving at the energies reported below.
We use these plots only to show the kind of repulsive Coulomb barrier that can be expected to "trap" the second electron from auto-detaching from $\mathrm{C}_{60}^{2-}$ and to show that $F(r)$ does not depend much on which form of the potential one uses.

Table I shows the mean-field and CI absolute energies (relative to $\mathrm{C}_{60}+e^{-}+e^{-}$) obtained for

TABLE I

Mean-field and $\mathrm{Cl}$ energies (eV), $\mathrm{Cl}$ wave function amplitudes $A$ and $B$, corresponding second-electron binding energies $\mathrm{BE}(\mathrm{eV})$, and correlation energies $E_{\text {Correl. }}(\mathrm{eV})$ for various radius parameters $r_{c^{*}}$

\begin{tabular}{rcccccr}
\hline$r_{c}$ (bohrs) & $E_{\mathrm{MF}}$ & $E_{\mathrm{Cl}}$ & $A / B$ & $B E_{\mathrm{MF}}$ & $B E_{\mathrm{Cl}}$ & $E_{\text {Correl. }}$ \\
\hline 7 & -1.97 & -2.24 & $0.95 / 0.32$ & -0.68 & -0.41 & -0.27 \\
8 & -2.31 & -2.60 & $0.92 / 0.40$ & -0.34 & -0.05 & -0.29 \\
9 & -2.60 & -2.90 & $0.91 / 0.40$ & -0.05 & +0.25 & -0.30 \\
10 & -2.85 & -3.15 & $0.90 / 0.44$ & +0.20 & +0.55 & -0.30 \\
\hline
\end{tabular}


$\mathrm{C}_{60}^{2-}$, as well as the second-electron binding energies predicted by these findings computed as the difference between the energy of $C_{60}^{2-}$ and $-2.65 \mathrm{eV}$ (the energy of $\mathrm{C}_{60}^{-}$). We also give $A$ and $B$ amplitudes corresponding to the $\mathrm{CI}$ wave function as well as the angular correlation energy $\left(E_{\mathrm{CI}}-E_{\mathrm{MF}}\right)$ for each value of radius parameter $r_{c}$.

Much as was found in Ref. [1], whose workers carried out a mean-field level calculation using the same kind of pseudo-potential as we employed, one has to characterize the framework of the $\mathrm{C}_{60}^{2-}$ dianion with a size near $r_{c}=10 \mathrm{bohrs}$ to realize a mean-field energy that is lower than the energy of $\mathrm{C}_{60}^{-}$. In our opinion, this is probably an unrealistic value for $r_{c}$. In contrast, when angular correlation is included in the calculation as we have done here, a value near $r_{c}=8$ bohrs renders $\mathrm{C}_{60}^{2-}$ barely stable relative to $\mathrm{C}_{60}^{-}+e^{-}$, whereas a value of $r_{c}=7$ bohrs predicts $C_{60}^{2-}$ to be unstable by $0.4 \mathrm{eV}$, the latter result being in good agreement with what the findings of Refs. [3-6] suggest. As mentioned earlier, the molecular framework of $\mathrm{C}_{60}$ is thought to have a radius a bit larger than $3.5 \AA=6.6$ bohrs, so we believe that the data listed in Table I for $r_{c}$ in the 7-8-bohr range are the most likely to be accurate.

The data in Table I also show clearly that the strength of the configuration mixing, as evidenced by the $B / A$ ratios, is considerable. We therefore suggest that any future ab initio calculations on this singlet state of $C_{60}^{2-}$ should take into consideration the multiconfigurational nature of the wave function.

Another observation we consider useful regarding the data presented in Table I is that the stabilizing angular correlation contribution to the energy of $\mathrm{C}_{60}^{2-}$ appears to be rather independent of $r_{c}$ and to be $\sim 0.3 \mathrm{eV}$. Keeping in mind that we used a variational approach for computing this correlation energy, any improvement in angular character of the trial wave function (e.g., using more terms in the expansion) will make the correlation energy even larger. Thus, we feel confident in concluding that angular correlation of the two "extra" electrons in $\mathrm{C}_{60}^{2-}$ gives stabilization (relative to a mean-field treatment) of $\geq 0.3 \mathrm{eV}$, which is probably the most important quantitative finding of this work.

As noted above (see column 6 of Table I), for values of $r_{c}$ in the (reasonable) 7-8-bohr range, singlet $C_{60}^{2-}$ is predicted, even when stabilizing angular correlation is considered, to be unstable by $0.05-0.4 \mathrm{eV}$. Because no radial correlation was in- cluded in our treatment, ${ }^{4}$ it is likely that singlet $C_{60}^{2-}$ is even a bit more stable than the latter numbers suggest. From Figure 1, it is clear that the Coulomb barriers through which an electron would have to tunnel to produce $\mathrm{C}_{60}^{-}+\mathrm{e}^{-}$are $\sim 1 \mathrm{eV}$ in height and are very broad, as is typical of such $e^{2} / r$ barriers. As pointed out in Ref. [4], there are also angular momentum contributions to the barrier caused by the fact that the excess electrons occupy orbitals of nonzero angular momentum, so the barriers through which tunneling must occur are even higher than in Figure 1.

Before closing, it is important to return briefly to the issue of whether a triplet state could, when the Compton-type potential is used, be lower in energy than the singlet state we have been focusing on in this discussion. Using the radial and angular functions introduced earlier, we can compute the energy of the triplet single-determinant wave function $\mid Y_{0,0}\left(\theta_{1}, \phi_{1}\right) F\left(r_{1}\right) / r_{1} \alpha_{1} Y_{1,0}\left(\theta_{2}, \alpha_{2}\right)$ $F\left(r_{2}\right) / r_{2} \alpha_{2} \mid$. For $r_{c}=7$ bohrs, we obtain -1.87 $\mathrm{eV}$, which is higher than the mean-field energy of the singlet state (see column 2 of Table I). Using $r_{c}=8$ bohrs, we obtain a triple mean-field energy of $-2.39 \mathrm{eV}$, which is slightly lower than the mean-field energy of the singlet state (see column 2 of Table I). Experience tells us that the kind of singlet state we studied here, in which a pair of electrons occupies the same orbital in the meanfield picture, has a larger correlation energy than does the triplet state, in which the electron occupy different orbitals. Therefore, it seems most likely that the singlet state, when angular correlations are included as is done here, will be the lower energy state of $\mathrm{C}_{60}^{2-}$.

\section{Summary}

Our findings suggest that singlet $\mathrm{C}_{60}^{2-}$ has an energy a bit less (because radial correlation is expected to lower the energy even below what we obtained) than $0.4 \mathrm{eV}$ above $\mathrm{C}_{60}^{-}+e^{-}$, but Coulomb and angular barriers ${ }^{5}$ of $\geq 1 \mathrm{eV}$ in height cause this dianion to be metastable and to have a long life-

\footnotetext{
${ }^{4}$ To allow for radial correlation, we could include in our variational wave function terms of the form $\mid Y_{0,0}\left(\theta_{1}, \phi_{1}\right) F\left(r_{1}\right) / r_{1} \alpha_{1}$ $Y_{0,-0}\left(\theta_{2}, \phi_{2}\right) F^{\prime}\left(r_{2}\right) / r_{2} \beta_{2} \mid$, in which the two electrons occupy different radial functions ( $F$ and $F^{\prime}$ ).

${ }^{5}$ Both Coulomb and angular momentum barriers arise in our CI simulation through the presence of the $e^{2} / r_{1,2}$ potential in the Hamiltonian and the $Y_{1,0}$ contributions to the trial wave functions.
} 
time. Although a triplet state of $C_{60}^{2-}$ may be close in energy to the singlet state we examined here, it seems likely that the singlet rather than the triplet is the lower-energy state. Moreover, our treatment of the angular correlations between the two excess electrons produces an estimate of $0.3 \mathrm{eV}$ for the corresponding correlation energy. Because this value is rather independent of the "size" parameter used to characterize the $\mathrm{C}_{60}$ molecular framework, it likely is a value that can be used by future workers to estimate such stabilizing contributions.

\section{ACKNOWLEDGMENTS}

The authors are grateful for significant computer time provided by the Center for High Performance Computing at the University of Utah.

\section{References}

1. Hettich, R. L.; Compton, R. N.; Ritchie, R. H. Phys Rev Lett 1991, 67, 1242.

2. Compton, R. N.; Tuinman, A. A.; Klots, C. E.; Pederson, M. R.; Patton, D. C. Phys Rev Lett 1997, 78, 4367.

3. (a) Chang, A. H. H.; Ermler, W. C.; Pitzer, R. M. J Phys Chem 1991, 95, 9288; (b) Scuseria, G. E. Chem Phys Lett 1991, 176, 423.

4. Yannouleas, C.; Landman, U. Chem Phys Lett 1994, 217, 175.

5. Martin, R. L.; Ritchie, J. P. Phys Rev B 1993, 48, 4845.

6. (a) Pederson, M. R.; Quong, A. A. Phys Rev B 1992, 46, 13584; (b) Pederson, M. R.; Jackson, K. A. Phys Rev B 1990, 41, 7453; (c) Jackson, K. A.; Pederson, M. R. Phys Rev B 1990, 42, 3276.

7. Simons, J. An Introduction to Theoretical Chemistry; Cambridge University Press: Cambridge, UK, 2003; p 201. 